\title{
Invariants of the electromagnetic field
}

\author{
C. A. Escobar ${ }^{1}$ and L. F. Urrutia \\ Instituto de Ciencias Nucleares, Universidad Nacional Autónoma de México, \\ A. Postal 70-543, 04510 México D.F., México and \\ Facultad de Física, Pontificia Universidad Católica de Chile, Casilla 306, Santiago 22, Chile
}

We present a constructive proof that, in electrodynamics, all of the gauge-invariant Lorentz scalars and pseudoscalars can be expressed as functions of the quadratic ones.

\section{INTRODUCTION}

In the limit where the gravitational, weak and strong interactions are not relevant, all classical electric and magnetic phenomena among electrically charged particles can be understood in terms of Maxwell's equations

$$
\begin{aligned}
& \nabla \cdot \mathbf{B}=0, \quad \nabla \times \mathbf{E}+\frac{1}{c} \frac{\partial \mathbf{B}}{\partial t}=0, \\
& \nabla \cdot \mathbf{E}=4 \pi \rho, \quad \nabla \times \mathbf{B}-\frac{1}{c} \frac{\partial \mathbf{E}}{\partial t}=\frac{4 \pi}{c} \mathbf{J},
\end{aligned}
$$

plus Newton's equations together with the Lorentz force

$$
\mathbf{F}=q\left(\mathbf{E}+\frac{\mathbf{v}}{c} \times \mathbf{B}\right) .
$$

Here $\mathbf{E}=\left(E_{1}, E_{2}, E_{3}\right)$ is the electric field, $\mathbf{B}=\left(B_{1}, B_{2}, B_{3}\right)$ is the magnetic induction, $\rho$ is the charge density, $\mathbf{J}$ is the current density, $\mathbf{v}$ is the velocity of the particle having charge $q$ and $c$ is the invariant speed of light. The above set of equations yield charge conservation in the form of $\partial \rho / \partial t+\nabla \cdot \mathbf{J}=\mathbf{0}$. We are using the notation, conventions and Gaussian units according to Ref. 1.

The reformulation of Maxwell's equations, Eqs. (10) and (2), in terms of Lorentz tensors in a four-dimensional Minkowski space (with metric $\eta_{\mu \nu}=\operatorname{diag}(+,-,-,-)$ ) is much more than a simple matter of changing to a convenient notation. This is because it makes conceptually transparent the deep interplay that exists among electric and magnetic phenomena. In the framework of special relativity an event is labeled by the spatial coordinates $x^{i}(i=1,2,3)$ and the time $x^{0}=c t$ (scaled, for dimensional reasons, by the invariant velocity of light), which are unified into the contravariant vector $x^{\mu}, \mu=0,1,2,3$, that transformes as

$$
x^{\prime \mu}=\Lambda_{\nu}^{\mu} x^{\nu}, \quad \Lambda_{\alpha}^{\mu} \eta_{\mu \nu} \Lambda_{\beta}^{\nu}=\eta_{\alpha \beta},
$$

when going from an inertial frame $S$ to an inertial frame $S^{\prime}$. The coordinate-independent $4 \times 4$ matrix $\Lambda_{\nu}^{\mu}$ describes the Lorentz transformation (rotations and boosts) that relates the two frames. The unification of electricity and magnetism into ED proceeds in an analogous way by recognizing that, in every inertial frame, the electric field $\mathbf{E}$ and the magnetic induction $\mathbf{B}$ are the components of an antisymmetric two-index tensor $F^{\mu \nu}$, called the field strength, according to the following

$$
F^{0 i}=-E_{i}, \quad F^{i j}=-\epsilon^{i j k} B_{k}
$$

Here $\epsilon^{i j k}$ is the completely antisymmetric three-index tensor with $\epsilon^{123}=+1$. The sources (charges $\rho$ and currents $\mathbf{J}$ ) also get unified in the current four-vector $J^{\mu}=(c \rho, \mathbf{J})$. The transformation properties of the tensor $F^{\mu \nu}$ allows us to determine how the components $E_{i}^{\prime}, B_{j}^{\prime}$ look in terms of $E_{i}, B_{j}$ when going from $S$ to $S^{\prime}$. With the above conventions we can directly verify that Maxwell's equations, Eqs. (1) and (2), can be compactly written as

$$
\epsilon^{\mu \nu \rho \sigma} \partial_{\nu} F_{\rho \sigma}=0, \quad \partial_{\mu} F^{\mu \nu}=\frac{4 \pi}{c} J^{\nu},
$$

respectively. The tensor $\epsilon^{\alpha \beta \mu \nu}$ is completely antisymmetric in each pair of indices with the convention that $\epsilon^{0123}=+1$. The introduction of the standard potentials $\Phi$, $\mathbf{A}$, which are unified now in four-potential $A_{\mu}=(\Phi,-\mathbf{A})$ satisfying $F_{\mu \nu}=\partial_{\mu} A_{\nu}-\partial_{\nu} A_{\mu}$, solves the first relation in (6). Notice also that the gauge transformations are written now as $A_{\mu} \rightarrow A_{\mu}+\partial_{\mu} \Theta\left(x^{\alpha}\right)$, which leave the field strength invariant.

As usual, a covariant index $\mu$ is obtained by lowering a contravariant index $\nu$ with the metric $\eta_{\mu \nu}$. Contractions over covariant and a contravariant indexes preserve the tensorial character of the remaining expression. A notable 
class of tensors occurs when, after the contractions, there are no free indices left. In this case we have what is called a scalar quantity under Lorentz transformations, with the property of having the same value in every inertial frame. The concept of tensors under Lorentz transformations is generalized by defining pseudo-tensors as quantities whose transformation rule include an additional factor of $\operatorname{det}\left[\Lambda_{\nu}^{\mu}{ }_{\nu}\right]$. Contractions upon a pseudotensor produce another pseudotensor, and ultimately a pseudoscalar when there are no free indices left.

The tensorial character of Eqs. (6) guarantees that they adopt the same form in every inertial frame. Also, the tensor formulation of ED together with the knowledge of the corresponding field invariants is an essential ingredient in the construction of non linear extensions of the theory, as well as in the construction of the couplings of the electromagnetic field to the other basic interactions. These new interaction terms are encoded in extended gauge invariant Lagrangian densities, which are required to be Lorentz scalars constructed from the basic fields under consideration, among which $A_{\mu}$ and/or $F_{\mu \nu}$ will be included.

\section{THE FIELD INVARIANTS}

This note provides an explicit proof of the well-known statement that in electrodynamics all the gauge invariant Lorentz scalars and pseudoscalars that can be constructed from the tensors $F_{\mu \nu}, \eta_{\mu \nu}$ (or equivalently $\delta_{\nu}^{\mu}$ ) and $\epsilon_{\alpha \beta \mu \nu}$ can be ultimately expressed in terms of the quadratic ones

$$
F=F_{\mu \nu} F^{\mu \nu}, \quad G=F^{\alpha \beta} \tilde{F}_{\alpha \beta},
$$

where $\tilde{F}_{\alpha \beta}$ is the dual tensor satisfying

$$
\tilde{F}_{\alpha \beta}=\frac{1}{2} \epsilon_{\alpha \beta \mu \nu} F^{\mu \nu}, \quad \frac{1}{2} \epsilon_{\alpha \beta \mu \nu} \tilde{F}^{\mu \nu}=-F_{\alpha \beta} .
$$

The fact that the only quadratic invariants are those in Eq. (7) is proved in many text-books. ${ }^{1-5}$

Surprisingly, a search that included well-known graduate text-books in electrodynamics, produced no examples of a complete proof of the above statement. Among the many references related to non-linear electrodynamics, which correctly assume that the most general Lorentz-invariant Lagrangian that can be written is of the form $L(F, G)$, we found Ref.6, which gives some hints regarding a general proof. The difficulty in finding a detailed proof of this statement has provided the motivation for writing this note.

Before going into the details it will be useful to distinguish between two approaches to the full proof. The first approach, which we call an existence proof, proceeds along the following lines: since all invariants are combinations of $F_{\mu \nu}, \eta_{\mu \nu}$ and $\epsilon_{\alpha \beta \mu \nu}$ and we know how to reduce an even product of epsilons, the only remaining objects that we must encounter at the end of the complete reduction must be the two quadratic invariants. A similar argument can be given in terms of the $S O(3)$ vectors $E_{i}, B_{i}$, which are the components of $F_{\mu \nu}$, provided that one first realizes that the only Lorentz invariants that can be constructed from the $\mathrm{SO}(3)$ quadratical invariants $\mathbf{E}^{2}, \mathbf{B}^{2}, \mathbf{E} \cdot \mathbf{B}, \operatorname{are}^{2}-\mathbf{B}^{2}$ and $\mathbf{E} \cdot \mathbf{B} .^{7}$

The second approach, which we call a constructive proof, classifies all possible invariants and provides the corresponding algorithm to reduce each of them to the final functions of $F$ and $G$.

Let us emphasize that our proof is neither the shortest, nor the most elegant, but it has the following two virtues: (i) it is more than an existence proof, since it contains a constructive procedure in the sense described above and (ii) it can be followed and understood by anyone familiar with standard graduate-level electrodynamics such as at the level of Refs.1,2,3,4,5. with no additional knowledge of more advanced mathematical tools. For example, a shorter existence proof can be carried out by using two-component spinor notation together with the decomposition of $F_{\mu \nu}$ into its selfdual and anti-selfdual parts. ${ }^{8}$ Another existence proof can be based upon the Cayley-Sylvester theorem, that determines the number of independent eigenvalues of $F_{\mu \nu}$, which are calculated in Ref. 9 and shown to depend only upon $F$ and $G .^{10}$

We now will begin our construction. It is convenient to introduce the following matrix notation

$$
\begin{aligned}
& \mathbf{F}=\left[F_{\nu}^{\mu}\right], \quad \tilde{\mathbf{F}}=\left[\tilde{F}_{\nu}^{\mu}\right], \quad \mathbf{F}^{p}=\underbrace{\mathbf{F F} \ldots \mathbf{F}}_{\text {ptimes }}, \quad \mathbf{F}^{0}=\mathbf{I}, \\
& \mathbf{f}=\left[F^{\mu \nu}\right], \quad \mathbf{f}^{T}=-\mathbf{f}, \quad \mathbf{m}=\left[\eta_{\alpha \beta}\right], \quad \mathbf{m}^{T}=\mathbf{m},
\end{aligned}
$$

which imply $\mathbf{F}=\mathbf{f m}$. In the above, $\mathbf{I}$ is the $4 \times 4$ identity operator. Notice that $\operatorname{Tr}\left(\mathbf{F}^{2}\right)=-F$. In terms of the matrices with definite symmetry, the product $\mathbf{F}^{p}$ can be written as

$$
\mathbf{F}^{p}=\underbrace{(\mathbf{f m})(\mathbf{f m}) \ldots(\mathbf{f m})}_{\text {times }}
$$


in such a way that

$$
\left(\mathbf{F}^{p}\right)^{T}=(-1)^{p} \underbrace{(\mathbf{m f})(\mathbf{m f}) \ldots(\mathbf{m f})}_{p \text { times }}
$$

A convenient way of classifying all the scalars and pseudoscalars is by writing an invariant of order $n$ (even or odd) in the field strength as

$$
I^{(n)}=\underbrace{F^{\mu \nu} F^{\alpha \beta} \ldots F^{\kappa \lambda}}_{n \text { times }} I_{\mu \nu \alpha \beta \ldots \kappa \lambda}
$$

where $I_{\mu \nu \alpha \beta \ldots \kappa \lambda}$ is constructed from the only tensor and pseudotensor that are invariant under the proper Lorentz transformations: $\eta_{\mu \nu}$ and $\epsilon_{\alpha \beta \mu \nu}$. The proof proceeds in three steps, according to the number of epsilon factors that occur in $I_{\mu \nu \alpha \beta \ldots \kappa \lambda}$, and it is presented in Sections III IV and V. The appendix includes the basic recurrence relations that are required in the following discussion.

\section{THE CASE OF NO EPSILON FACTORS IN $I_{\mu \nu \alpha \beta \ldots \kappa \lambda}$}

Since $I_{\mu \nu \alpha \beta \ldots \kappa \lambda}$ is now constructed from the metric tensor only, a generic member of this class will have the form

$$
\operatorname{Tr}\left(\mathbf{F}^{p}\right) \operatorname{Tr}\left(\mathbf{F}^{q}\right) \ldots \operatorname{Tr}\left(\mathbf{F}^{r}\right)
$$

with $p+q+\ldots+r=n$, and where we have omitted contractions of the type $\eta_{\rho \sigma} F^{\rho \sigma}$ that have a zero contribution. In this way, for this case it is enough to consider the reduction of an arbitrary factor $\operatorname{Tr}\left(\mathbf{F}^{p}\right)$.

The antisymmetry of $\mathbf{f}$ guarantees that $\operatorname{Tr}\left(\mathbf{F}^{n}\right)=0$, for odd $n$. In fact, from Eq. (12) we have

$$
\operatorname{Tr}\left(\mathbf{F}^{n}\right)=\operatorname{Tr}\left(\left(\mathbf{F}^{n}\right)^{T}\right)=\underbrace{-\operatorname{Tr}[(\mathbf{m f})(\mathbf{m f}) \ldots(\mathbf{m f})]}_{n \text { times }}=\underbrace{-\operatorname{Tr}[(\mathbf{f m})(\mathbf{f m}) \ldots(\mathbf{f m})]}_{n \text { times }}=-\operatorname{Tr}\left(\mathbf{F}^{n}\right)=0
$$

by using the cyclic property of the trace.

The case of an even $p$ can be reduced by using a recurrence relation, Eq. (A7), which ultimately produces

$$
\begin{aligned}
\operatorname{Tr}\left(\mathbf{F}^{p}\right)= & -\frac{F}{2} \operatorname{Tr}\left(\mathbf{F}^{p-2}\right)+\frac{G^{2}}{16} \operatorname{Tr}\left(\mathbf{F}^{p-4}\right) \\
& \ldots \ldots, \\
= & U\left(F, G^{2}\right) \operatorname{Tr}\left(\mathbf{F}^{2}\right)+V\left(F, G^{2}\right) \operatorname{Tr}\left(\mathbf{F}^{0}\right) \\
= & -U\left(F, G^{2}\right) F+4 V\left(F, G^{2}\right)
\end{aligned}
$$

where $U, V$ denote the functions of the quadratic invariants that appear in the reduction process. When similar functions appear below, an analogous notation will be used without stating this fact at each step.

In this way, repeated use of Eq. (A7) allows one to reduce $\operatorname{Tr}\left(\mathbf{F}^{p}\right)$ to a function of $F$ and $G$, where $G$ appears only in even powers, which is due to parity conservation.

\section{THE CASE OF AN EVEN NUMBER OF EPSILON FACTORS IN $I_{\mu \nu \alpha \beta \ldots \kappa \lambda}$}

Using Eq. A3 as many times as required, we first reduce all pairs of epsilons, independently of how they are contracted, and in this way this case is transformed into that discussed in Section III.

\section{THE CASE OF AN ODD NUMBER OF EPSILON FACTORS IN $I_{\mu \nu \alpha \beta \ldots \kappa \lambda}$}

The use of Eq. (A33) allows us to reduce this case to the situation where $I_{\mu \nu \alpha \beta \ldots \kappa \lambda}$ has only one epsilon factor, and all the remaining pairs have been written in terms of products of the metric tensor. We then need to consider only a generic invariant of order $n$ with

$$
I_{\mu \nu \ldots \alpha \beta \kappa \lambda \pi \delta}^{(n)}=\underbrace{\eta_{\mu \nu \cdots \eta_{\alpha \beta}}}_{n-2 \text { times }} \epsilon_{\kappa \lambda \pi \delta}
$$


Since the indices in $F^{\alpha \beta}$ come in pairs the only form of an invariant containing only one epsilon factor is

$$
I^{(n)}=\operatorname{Tr}\left(\mathbf{F}^{p}\right)\left(\mathbf{F}^{q}\right)^{\kappa \lambda}\left(\mathbf{F}^{r}\right)^{\pi \delta} \epsilon_{\kappa \lambda \pi \delta},
$$

where $p+q+r=n$. The trace part is reduced according to Section III and we only need to consider the remaining invariant

$$
I^{(q+r)}=\left(\mathbf{F}^{q}\right)^{\kappa \lambda} \epsilon_{\kappa \lambda \pi \delta}\left(\mathbf{F}^{r}\right)^{\pi \delta}
$$

Here the reduction is performed by using the recurrence relations of Eqs. (A7), (A9), and (A10) for each factor of $\left(\mathbf{F}^{m}\right)^{\kappa \lambda}$ that appears Eq. (19). The intermediate results are

$$
\begin{aligned}
& \mathbf{F}^{m}=W\left(F, G^{2}\right) \mathbf{I}+Z\left(F, G^{2}\right) \mathbf{F}^{2}, \quad m \text { even } \\
& \mathbf{F}^{m}=\bar{Z}\left(F, G^{2}\right) \mathbf{F}+\bar{W}\left(F, G^{2}\right) G \tilde{\mathbf{F}}, \quad m \text { odd },
\end{aligned}
$$

which yield the final four possibilities

$$
\begin{aligned}
& I_{q \text { even, } r \text { even }}^{(q+r)}=\left(\mathbf{F}^{2}\right)^{\kappa \lambda} \epsilon_{\kappa \lambda \pi \delta}\left(\mathbf{F}^{2}\right)^{\pi \delta} \bar{Z}_{1}, \\
& I_{q \text { even }, r \text { odd }}^{(q+r)}=\left(\mathbf{F}^{2}\right)^{\kappa \lambda} \epsilon_{\kappa \lambda \pi \delta} F^{\pi \delta} \bar{Z}_{2}+\left(\mathbf{F}^{2}\right)^{\kappa \lambda} \epsilon_{\kappa \lambda \pi \delta} \tilde{F}^{\pi \delta} \bar{Z}_{3}, \\
& I_{q \text { odd, r even }}^{(q+r)}=F^{\kappa \lambda} \epsilon_{\kappa \lambda \pi \delta}\left(\mathbf{F}^{2}\right)^{\pi \delta} \bar{Z}_{4}+\tilde{F}^{\kappa \lambda} \epsilon_{\kappa \lambda \pi \delta}\left(\mathbf{F}^{2}\right)^{\pi \delta} \bar{Z}_{5}, \\
& I_{q \text { odd, } r \text { odd }}^{(q+r)}=\tilde{F}^{\kappa \lambda} \epsilon_{\kappa \lambda \pi \delta} \tilde{F}^{\pi \delta} \bar{Z}_{6}+\tilde{F}^{\kappa \lambda} \epsilon_{\kappa \lambda \pi \delta} F^{\pi \delta} \bar{Z}_{7}+F^{\kappa \lambda} \epsilon_{\kappa \lambda \pi \delta} F^{\pi \delta} \bar{Z}_{8},
\end{aligned}
$$

where $\bar{Z}_{A}, A=1,2,3, \ldots, 8$ are already functions of $F$ and $G$. The final reduction of the terms involved above proceeds as follows. Equation (22) yields

$$
\left(\mathbf{F}^{2}\right)^{\kappa \lambda} \epsilon_{\kappa \lambda \pi \delta}\left(\mathbf{F}^{2}\right)^{\pi \delta}=0
$$

because $\left(\mathbf{F}^{2}\right)^{\kappa \lambda}=\left(\mathbf{F}^{2}\right)^{\lambda \kappa}$. The reduction of Eqs. (23) and (24) include the same terms and have two contributions that yield

$$
\left(\mathbf{F}^{2}\right)^{\kappa \lambda} \epsilon_{\kappa \lambda \pi \delta} F^{\pi \delta}=0, \quad \tilde{F}^{\kappa \lambda} \epsilon_{\kappa \lambda \pi \delta}\left(\mathbf{F}^{2}\right)^{\pi \delta}=0
$$

again due to the symmetric character of $\left(\mathbf{F}^{2}\right)^{\kappa \lambda}$. The last reduction arises from Eq. (25) which includes

$$
\tilde{F}^{\kappa \lambda} \epsilon_{\kappa \lambda \pi \delta} \tilde{F}^{\pi \delta}=-2 G \quad \tilde{F}^{\kappa \lambda} \epsilon_{\kappa \lambda \pi \delta} F^{\pi \delta}=-2 F \quad F^{\kappa \lambda} \epsilon_{\kappa \lambda \pi \delta} F^{\pi \delta}=2 G .
$$

Here we have made use of the second relation in Eq. (8) together with the definition of $G$. This completes the proof.

\section{ACKNOWLEDGEMENTS}

L.F.U is partially supported by the project DGPA-IN109013 and a sabbatical fellowship from DGAPA-UNAM. He also acknowledges the hospitality of the Facultad de Física and support from the Programa de Profesores Visitantes, at the Pontificia Universidad Católica de Chile. C.A.E acknowledges support from a CONACyT graduate fellowship as well as partial support from the project DGAPA-IN109013 and the program PAEP at UNAM. The authors gratefully acknowledge Dr. E. Nahmad and Dr. M. A. García for useful suggestions.

\section{Appendix A}

The basic relations employed in the proof are

$$
\begin{gathered}
F_{\lambda}^{\mu} F_{\nu}^{\lambda}=\tilde{F}_{\lambda}^{\mu} \tilde{F}_{\nu}^{\lambda}-\frac{1}{2} F \delta_{\nu}^{\mu}, \\
\tilde{F}_{\lambda}^{\mu} F_{\nu}^{\lambda}=-\frac{1}{4} G \delta_{\nu}^{\mu},
\end{gathered}
$$


which are well known. ${ }^{6,9,11,12}$ The first one is a direct consequence of the definition of the dual tensor together with the property

$$
\epsilon_{\mu \nu \rho \sigma} \epsilon_{\pi \delta \kappa \lambda}=\operatorname{det}\left[\begin{array}{llll}
\eta_{\mu \pi} & \eta_{\mu \delta} & \eta_{\mu \kappa} & \eta_{\mu \lambda} \\
\eta_{\nu \pi} & \eta_{\nu \delta} & \eta_{\nu \kappa} & \eta_{\nu \lambda} \\
\eta_{\rho \pi} & \eta_{\rho \delta} & \eta_{\rho \kappa} & \eta_{\rho \lambda} \\
\eta_{\sigma \pi} & \eta_{\sigma \delta} & \eta_{\sigma \kappa} & \eta_{\sigma \lambda}
\end{array}\right]
$$

Equation (A2) arises by parity arguments and the fact that the only quadratic invariant that violates parity is $G$. In this way, the Lorentz covariance demands that

$$
\tilde{F}_{\lambda}^{\mu} F_{\nu}^{\lambda}=\alpha G \delta_{\nu}^{\mu}
$$

where the factor $\alpha=-1 / 4$ is obtained by contracting Eq. (A4) with $\delta_{\mu}^{\nu}$ and using the definition of $G$.

It is now convenient to go back to the matrix notation introduced in Eq.(9) and rewrite Eqs. (A1) and (A2) as

$$
\begin{gathered}
\mathbf{F}^{2}=\tilde{\mathbf{F}}^{2}-\frac{1}{2} F \mathbf{I}, \\
\tilde{\mathbf{F}} \mathbf{F}=\mathbf{F} \tilde{\mathbf{F}}=-\frac{1}{4} G \mathbf{I} .
\end{gathered}
$$

The above equations allow us to write the following recursion relation

$$
\mathbf{F}^{p}=-\frac{F}{2} \mathbf{F}^{p-2}+\frac{G^{2}}{16} \mathbf{F}^{p-4}
$$

which is obtained as follows

$$
\begin{aligned}
\mathbf{F}^{p} & =\mathbf{F}^{p-2} \mathbf{F}^{2}=\mathbf{F}^{p-2} \tilde{\mathbf{F}}^{2}-\frac{1}{2} F \mathbf{F}^{p-2}, \\
\mathbf{F}^{p} & =\mathbf{F}^{p-4} \mathbf{F} \mathbf{F} \tilde{\mathbf{F}} \tilde{\mathbf{F}}-\frac{1}{2} F \mathbf{F}^{p-2}, \\
\mathbf{F}^{p} & =\frac{1}{16} G^{2} \mathbf{F}^{p-4}-\frac{1}{2} F \mathbf{F}^{p-2},
\end{aligned}
$$

where we have used Eq. A5 in the first line, together with Eq. (A6) twice in the second line. For an even $p$, the recurrence will end at $p=4$, leading to

$$
\mathbf{F}^{4}=\frac{1}{16} G^{2} \mathbf{I}-\frac{1}{2} F \mathbf{F}^{2} .
$$

In the case of an odd $p$, the final result will correspond to $p=3$

$$
\mathbf{F}^{3}=\mathbf{F}\left(\mathbf{F}^{2}\right)=\mathbf{F}\left(\tilde{\mathbf{F}} \tilde{\mathbf{F}}-\frac{1}{2} F \mathbf{I}\right)=-\frac{1}{4} G \tilde{\mathbf{F}}-\frac{1}{2} F \mathbf{F} .
$$

In an analogous way we can show that

$$
\tilde{\mathbf{F}}^{p}=\frac{1}{16} G^{2} \tilde{\mathbf{F}}^{p-4}+\frac{1}{2} F \tilde{\mathbf{F}}^{p-2}
$$

Again, some care must be taken in the final step of the above recurrence relation. We obtain

$$
\begin{array}{rlrl}
p \text { even : } & \tilde{\mathbf{F}}^{4} & =\left(\frac{1}{16} G^{2}+\frac{1}{4} F^{2}\right) \mathbf{I}+\frac{1}{2} F \mathbf{F}^{2}, \\
& p \text { odd : } & \tilde{\mathbf{F}}^{3} & =-\frac{1}{4} G \mathbf{F}+\frac{1}{2} F \tilde{\mathbf{F}} .
\end{array}
$$

Other useful relations that can be derived directly from the above are

$$
\begin{gathered}
\tilde{F}^{\alpha \beta} \epsilon_{\beta \mu \nu \rho}=\left(F_{\mu \nu} \delta_{\rho}^{\alpha}+F_{\nu \rho} \delta_{\mu}^{\alpha}+F_{\rho \mu} \delta_{\nu}^{\alpha}\right) \\
\mathbf{F}^{m} \tilde{\mathbf{F}}^{n}=\left(-\frac{G}{4}\right)^{n} \mathbf{F}^{m-n}, m>n, \quad \mathbf{F}^{m} \tilde{\mathbf{F}}^{n}=\left(-\frac{G}{4}\right)^{m} \tilde{\mathbf{F}}^{n-m}, m<n .
\end{gathered}
$$


[1] J. D. Jackson, Classical Electrodynamics, Third edition (John Wiley and Sons, New York, 1999).

[2] F. W. Hehl and Y. N. Obukhov, Foundations of Classical Electrodynamics (Birkhauser, Boston, 2003).

[3] L. D. Landau and E. M. Lifshitz, The Classical Theory of Fields, Fouth revised english version (Butterworth-Heinemann, Oxford, 2000).

[4] A. Sommerfeld, Electrodynamics (Academic Press Inc., New York, 1952).

[5] J. A. Stratton, Electromagnetic Theory (IEEE Press Series, Piscataway, New Jersey, 2007).

[6] W. Dittrich and H. Gies, Phys. Rev. D58, 025004(1998).

[7] A. Rebhan (private communication)

[8] W. Siegel (private communication)

[9] J. Schwinger, Phys. Rev. 82, 664 (1951).

[10] S. Deser (private communication)

[11] V. A. De Lorenci, R. Klippert, M. Novello and J. M. Salim, Phys. Lett. B 482,134 (2000).

[12] M. Novello, V. A. De Lorenci, J. M. Salim and R. Klippert, Phys. Rev. D 61, 045001 (2000). 